\title{
FROG characterisation of SOA-based wavelength conversion using XPM in conjunction with shifted filtering up to line rates of $80 \mathrm{GHz}$
}

\author{
A.M. Clarke ${ }^{1}$, G. Girault ${ }^{2}$, P. Anandarajah ${ }^{1}$, C. Guignard ${ }^{1}$, L. Bramerie ${ }^{2}$, L.P. Barry ${ }^{1}$, J. C. Simon ${ }^{2}$ and J. Harvey ${ }^{3}$ \\ ${ }^{1}$ RINCE, School of Electronic Eng., Dublin City University, Ireland (email:liam.barry@dcu.ie). \\ ${ }^{2}$ FOTON-ENSSAT / PERSYST Platform, 6 rue de Kérampont, BP 8051822305 Lannion Cedex, France. \\ ${ }_{3}$ Physics Dept. Auckland University, New Zealand.
}

Semiconductor Optical Amplifiers (SOAs) are ideal candidates for use as all-optical processing devices such as wavelength conversion [1]. Their attraction comes from their small size, low energy consumption, highly nonlinear response and possibilities for monolithic integration. The work we present here builds on recent work where we obtained $80 \mathrm{~Gb} / \mathrm{s}$ error free performance using cross phase modulation (XPM) in an SOA in conjunction with a blue shifted bandpass filter [2]. Here we present a detailed characterisation of this wavelength conversion scheme using a Frequency Resolved Optical Gating (FROG) measurement scheme for both red and blue shifted filtering. This characterisation is important as it measures the exact temporal profile of the wavelength converted pulses and its corresponding output chirp as a function of input line rate from 10-80 GHz. Thus the patterning effect and the gain dynamics resulting from red and blue shifted filtering can be examined in detail. The FROG measurement scheme is advantageous over other pulse measurement schemes such as bandwidth limited oscilloscopes and traditional autocorrelation methods as it retrieves intensity and corresponding phase information [3].

The wavelength conversion scheme presented here uses a very simple technique, which exhibits low polarisation dependence and retains the polarity of the input pulse in comparison to other similarly published wavelength conversion schemes [4]. The experimental set-up is shown in Figure 1. Mode-locked pulses with a FWHM of 2.3 ps were generated at a repetition rate of $10 \mathrm{GHz}$ at a wavelength of $1545 \mathrm{~nm}$. The pulses were amplified and passively multiplexed at rates ranging from $10-80 \mathrm{GHz}$. This pulse train was coupled with a continuous wave (CW) probe signal and were injected into a commercially available SOA. The measured slow gain recovery time of this SOA was $50 \mathrm{ps}$. The pulse energy was kept constant for each line rate at $12 \mathrm{fJ}$ and the probe average power was $2.5 \mathrm{dBm}$. To obtain optimised red and blue shifted filtering the probe signal was set at a wavelength of $1551.91 \mathrm{~nm}$ and $1558.6 \mathrm{~nm}$ respectively. The probe signal was tuned as a fixed Fibre Bragg Grating Filter was used following the SOA. This filter had a $3 \mathrm{~dB}$ bandwidth of $5 \mathrm{~nm}$ and a sharp band edge, which was used to reject the original pump signal and to significantly reduce the $\mathrm{CW}$ portion of the probe. A second $3 \mathrm{~nm}$ tunable Gaussian filter was used to suppress further the $\mathrm{CW}$ signal and thus give an improved extinction ratio (ER). The intermediate EDFA (EDFA2) was used to overcome the high insertion loss due to the shifted filters. The wavelength converted pulses were then analysed using the FROG at line rates of 10, 20, 40, and $80 \mathrm{GHz}$. An EDFA was required before the FROG to obtain the maximum SNR of the measurement scheme.

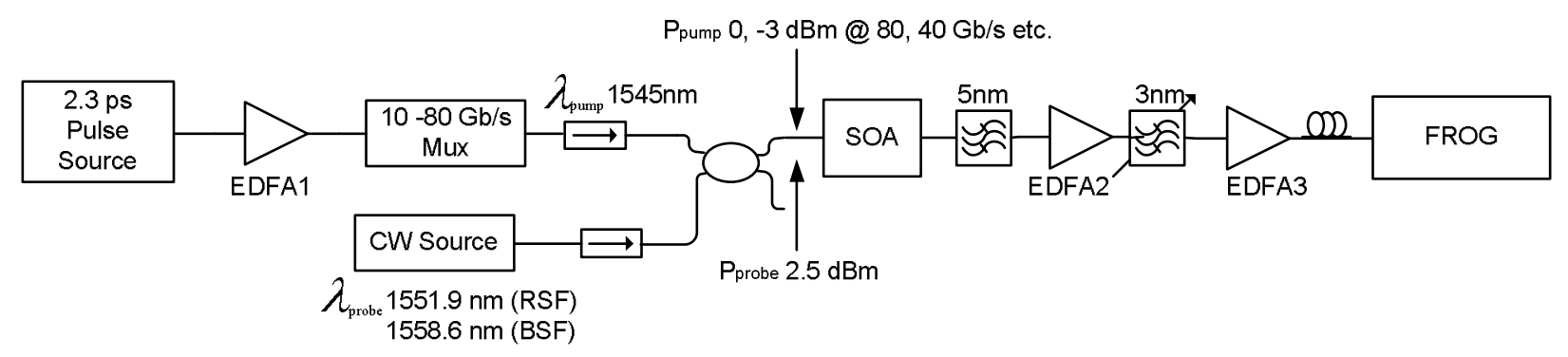

Figure 1: Experimental set up for the bandpass shifted filtering wavelength conversion scheme

The intensity profiles of the wavelength converted pulses for red and blue shifted filtering are shown in Figure 2. By placing the filter on the red shifted side of the original probe spectrum we obtain very short pulses as shown in Figure 2(a). This is due to the very fast response caused by the depletion of carriers which induces an equally fast red chirp. A small portion of blue chirped spectral components, which are not fully rejected by the filters, is the cause of the tail present on the trailing edge of the pulses. As is clearly evident, patterning effects are very prominent in red shifted wavelength conversion schemes, as the amount of carriers depleted dictates the size of the chirp and as the bit rates increase, fewer carriers have recovered. This leads to a reduction in red chirp and thus a reduction in output power. However, for the blue shifted filtering we can obtain error free performance up to $80 \mathrm{~Gb} / \mathrm{s}$ [2] as the patterning effects are not as substantial as displayed in Figure 2(b), as blue shifted filtering is less dependent on the 
input line rate. The leading edges of the blue shifted wavelength converted pulses have a very sharp slope. This is the result of the fast gain recovery of the SOA, which is related to intraband effects occurring on a timescale of approx 1-2ps. The structure at the centre of the pulse is due to a combination of the filter shape and the point where the slow gain recovery begins to take effect. The trailing edge of the pulse is determined by the shape of the filter profile.
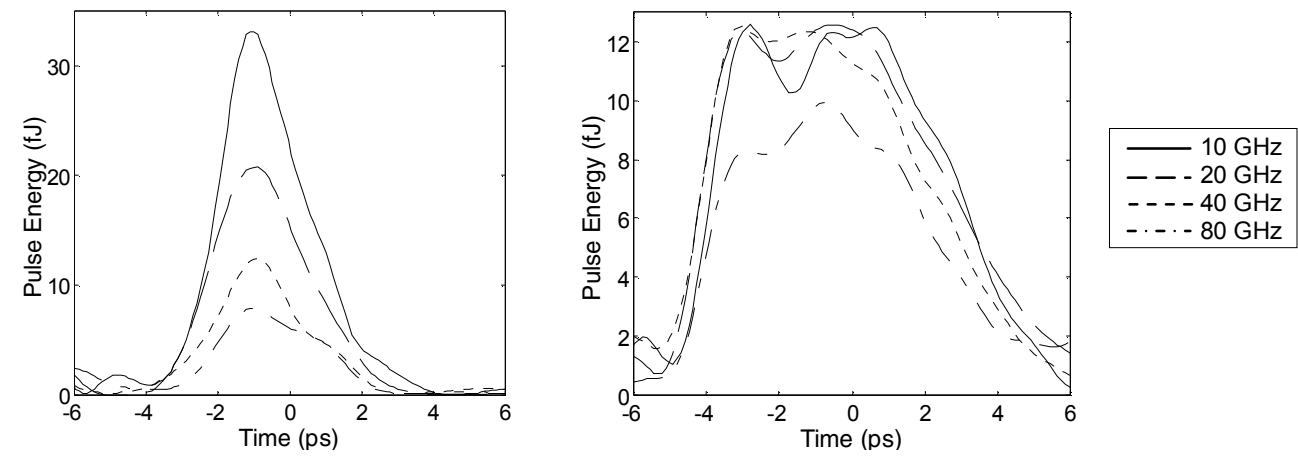

Figure 2 (a) and (b): Intensity profiles for red and blue shifted filtering respectively in conjunction with XPM in an SOA at line rates of $10,20,40$ and $80 \mathrm{GHz}$.

In Figure 3 we show the wavelength converted pulses normalised and their corresponding output chirp. The output pulses have a pulse width of 2.5-3.6 ps and 7-6.3 ps for respective red and blue shifted converted pulses. The chirp in particular for the blue shifted pulses is approximately linear across the pulse. This is to be expected as the induced phase by the SOA is converted into amplitude modulation by the shifted filter. This linear chirp with an approximate magnitude of $200 \mathrm{GHz}$ could be used to compress the blue-shifted converted pulses to $3.5 \mathrm{ps}$. The small ripple on the output chirp is due to an uneven filter profile. Enhanced performance could be achieved by using a filter with a smoother profile and pulse compression could be obtained with a filter that has a linear chirp opposite to the wavelength converted pulses.
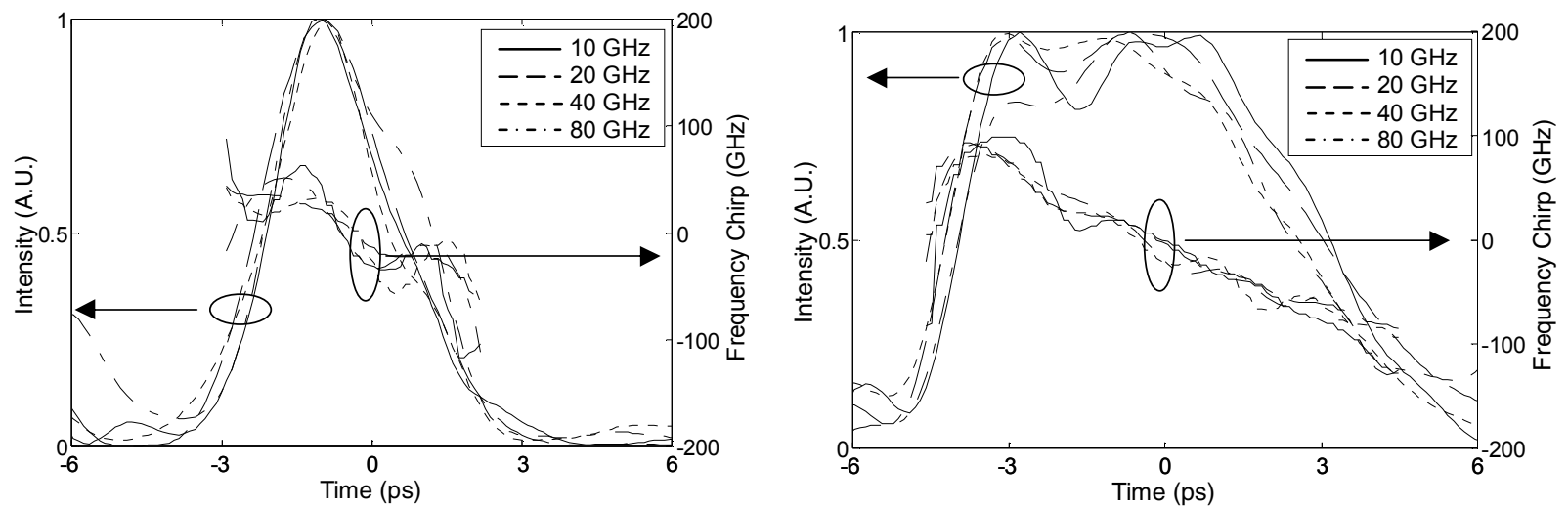

Figure 3 (a) and (b): Intensity and corresponding chirp profiles for red and blue shifted filtering respectively in conjunction with XPM in an SOA at line rates of $10,20,40$ and $80 \mathrm{GHz}$.

In conclusion a full characterisation of a popular wavelength conversion scheme which uses XPM in an SOA in conjunction with bandpass shifted filtering is provided. This type of characterisation has not been provided before to the best of the authors knowledge and is an important analysis firstly to achieve a full understanding of the gain and phase dynamics exploited by the wavelength conversion scheme presented and secondly to design a filter so that an optimum performance can be obtained.

Acknowledgements: This work was supported in part by Science Foundation Ireland (Investigator Program), the French Research Ministry, Europe (FEDER) and Britanny Region.

[1] T. Durhuus et al., IEEE J. of Lightwave Technol., vol. 14, pp. 942-954, 1996.

[2] L. Bramerie et al., CLEO 2006, CMT7, 2006.

[3] R. Trebino, et al., Rev. Sci. Instrum., vol. 68, pp. 3277-3295, 1997.

[4] Y. Liu, et al., IEEE J. of Lightwave Technol., vol. 24, pp. 230-236, 2006. 